\title{
POTENCIAL ACUÍCOLA DE LOS RECURSOS HÍDRICOS CONTINENTALES DE LA REGIÓN TACNA
}

\section{RESUMEN}

En el presente trabajo de Investigación se determinó el potencial o aptitud acuícola de un total de treinta (30) recursos hídricos continentales de la región Tacna, de los cuales 22 son ambientes lóticos (ríos) y 08 ambientes lénticos (lagunas y represas). El potencial acuícola de cada uno de los recursos hídricos se estimó según un estudio limnológico (características físicas, químicas y biológicas). La mayoría de recursos hídricos evaluados presenta posibilidades para el desarrollo de actividades acuícolas de repoblamiento y cultivo semiintensivo e intensivo de trucha arco iris Oncorhynchus mykiss y de camarón nativo Cryphiops caementarius. Los únicos recursos hídricos en los cuales no es posible el establecimiento de estos programas son el río Tarucachi, ubicado en la provincia de Tarata, y el río Caplina, ubicado en la provincia de Tacna, por la mala calidad de sus aguas, especialmente por su acidez ( $\mathrm{pH}$ menos de 4,5) y la laguna Vilacota, por ser un ambiente muy somero. Los programas de truchicultura se pueden implementar o afianzar en aquellos recursos hídricos cuya cuenca esté por encima de los 2500 msnm, y los de camarón en aquellos recursos hídricos cuya cuenca esté por debajo de esa altitud.

\begin{abstract}
In the present Work of Investigation the potential or acuícola aptitude of a total of thirty (30) continental hydric resources of the Tacna region was determined, of which 22 are ambient lóticos (rivers) and 08 lénticos (lagoons and dams). The acuicola potential of each one of the hydric resources was considered on the basis of a limnológico study (characteristic physical, chemical and biological). Most of evaluated hydric resources it presents/displays possibilities for the development of acuicolas activities of repoblamiento and semi-intensive and intensive culture of trout rainbow Oncorhynchus mykiss and native shrimp Cryphiops caementarius. The only hydric resources in which the establishment of these programs is not possible are the Tarucachi river, located in the province of Tarata and the Caplina river, located in the province of Tacna, by the bad quality of their waters, specially by its acidity ( $\mathrm{pH}$ less than 4.5) and the Vilacota lagoon, for being a very brief ambient. The truchicultura programs can be implemented or be strengthened in those hydric resources whose river basin is over $2500 \mathrm{msnm}$, whereas those of shrimp in those hydric resources whose river basin is below that altitude.
\end{abstract}

\section{INTRODUCCION}

Los recursos hidricos de la región Tacna son muy escasos si se comparan con los existentes en otras regiones, principalmente los de la sierra y selva, debido a su posición geográfica en que se encuentra la zona en relación con su ubicación en el globo terráqueo. Nos encontramos en una zona subtropical de características desérticas por excelencia, de allí la escasez de recursos hídricos y de recursos hidrobiológicos continentales.

Además de ser escasos en número, los recursos lóticos o ríos acarrean una limitada cantidad de agua con periodicidad temporal, que disminuye grandemente en épocas de estiaje. Los recursos lénticos o lagunas de igual modo son muy limitados en número, se ubican casi en su totalidad en la parte alta de la Cordillera Occidental, la misma que constituye el inicio de la zona altiplánica.
Los recursos lóticos de la región y evaluados en el presente estudio, se los ha agrupado en cuatro cuencas hidrográficas: Locumba (ríos Huaytire, Tacalaya, Callazas, Salado, Curibaya, llabaya y Locumba, manantial Chaullapujo y Quebrada Honda), Sama (ríos Ticalaco, Tarucachi, Pistala, Chucatamani, Salado y Sama), Caplina-Uchusuma (ríos Uchusuma y Caplina) y Maure (ríos Maure, Paucarani, Condorpico y Caño).

Los recursos lénticos o lagunas están conformados por ambientes permanentes y temporales. En el presente estudio, se han evaluado los siguientes ambientes lénticos: Laguna Suches, Laguna Aricota, Laguna Condorpico, Laguna Casiri, Laguna Vilacota, Represa Jarumas, Represa Paucarani y Represa Pampa de Vaca. La mayoría de estos ambientes acuáticos se encuentran sobre los $4000 \mathrm{msnm}$. La Laguna Aricota y la Represa Pampa de Vaca están situadas a menor altitud (2800 y 3900 msnm, respectivamente). 
Además de los recursos lénticos mencionados, existen en la zona represas, tales como las de Jarumas y Paucarani. Estas represas todavía no están siendo aprovechadas integralmente, aunque en la represa de Jarumas se viene cultivando en forma extensiva (repoblamiento) trucha arco iris por parte de la "Asociación de Extractores de Trucha de la Represa Jarumas-Anexo Mamaraya".

El objetivo principal del estudio fue determinar y precisar los recursos hídricos continentales de la región Tacna, factibles de ser utilizados en el cultivo de peces y crustáceos, con la finalidad de lograr un aprovechamiento integral de los escasos recursos hídricos con los que cuenta la región.

\section{MATERIALYMETODOS}

1.Muestreos: Para los trabajos de campo se fijaron Estaciones de Muestreo tomando en cuenta dos niveles de altitud de las cuencas hidrográficas: el primero a nivel de costa (por debajo de los $2000 \mathrm{msnm}$ ) y el segundo a nivel de la región altoandina (sobre los $2000 \mathrm{msnm}$ ). Además, dichas estaciones de muestreo fueron ubicadas en lugares de fácil accesibilidad y próximas a las vías de comunicación.

En cada una de las estaciones se realizaron las siguientes determinaciones: Caudal del río, tipología del cauce, determinación de parámetros físicos (temperatura del agua, transparencia del agua y coloración), químicos (concentra-ciones de oxígeno disuelto, anhídrido carbónico libre, alcalinidad, potencial de hidrogeniones, dureza y aniones y cationes presentes en el agua y biológicos: fitoplancton, macroinvertebrados, microin-vertebrados (zooplancton), fitobentos y macrofitas acuáticas).

2. Materiales y Métodos: Para la determinación de los diferentes parámetros físico-químicos y biológicos de los recursos hídricos considerados en el estudio, se ha hecho uso de materiales, equipos y meto-dología estandarizada y de uso común y corriente para este tipo de estudios limnológicos.

Algunos parámetros físico-químicos fueron determinados "in situ", tales como por ejemplo: temperatura, transparencia, coloración, gases (oxígeno disuelto y anhídrido carbónico libre), alcalinidad y dureza, utilizando un Laboratorio portátil Marca $\mathrm{HACH}$ Modelo FF-2. Análisis químicos y biológicos más completos fueron realizados en los Laboratorios de Tecnología Pesquera y de Ecología, respectivamente de la Facultad de Ingenieria Pesquera de la Universidad Nacional Jorge Basadre Grohmann de Tacna.

\section{RESULTADOSYDISCUSION}

\section{Potencial o Aptitud Acuícola de la Cuenca} del Río Sama

El potencial o aptitud acuícola de los recursos hídricos de la cuenca del río Sama evaluados en el presente estudio y determinado según los índices o unidades de productividad, se detallan en el Cuadro 1.

Cuadro 01: Aptitud acuícola de los recursos hídricos de la cuenca del río Sama.

\begin{tabular}{|c|c|c|c|c|}
\hline$\omega^{*}$ & RECURSO HIDRICD & $\begin{array}{l}\text { APTITUD } \\
\text { ACUICOLA }\end{array}$ & $\begin{array}{l}\text { RECURSO } \\
\text { HOROBIOLOGICO }\end{array}$ & $\begin{array}{l}\text { ACTIMDAND } \\
\text { ACUICOLA }\end{array}$ \\
\hline 1 & Rio Sams (Corvca) & & Camarón, trucha & $\begin{array}{l}\text { Repoblamiento, } \\
\text { cultivo }\end{array}$ \\
\hline 2 & Rlo sama (tala) & Medlana & Trucha & $\begin{array}{l}\text { Repoblamiento, } \\
\text { cultivo }\end{array}$ \\
\hline 3 & Rio Tiealieco & Mediana & Trucha & $\begin{array}{l}\text { Repoblamiento, } \\
\text { cultivo }\end{array}$ \\
\hline 4 & Rio Saludo & Mediana & Trucha & $\begin{array}{l}\text { Repoblamiento, } \\
\text { cultivo }\end{array}$ \\
\hline 5 & Rio Pistula & Baja & Trucha & $\begin{array}{l}\text { Repoblamiento, } \\
\text { cultivo }\end{array}$ \\
\hline 6 & Rio Chueatamani & Baja & Trucha & $\begin{array}{l}\text { Repoblaniento, } \\
\text { cultivo }\end{array}$ \\
\hline 7 & Rio Terrucach & tots & Ninguno & \begin{tabular}{|l|} 
Ninguna \\
\end{tabular} \\
\hline
\end{tabular}

De toda la cuenca del río Sama, este río en la zona de Coruca es el que ostenta la más alta productividad natural y, por consiguiente, la mejor aptitud acuícola para el establecimiento de programas para el cultivo extensivo (repoblamiento) e intensivo (cultivo) de camarón de río Cryphiops caementarius.

\section{Potencial o Aptitud Acuícola de la Cuenca del Río Locumba}

El potencial o aptitud acuícola de los recursos hídricos (rios, manantiales y quebradas) de la cuenca del río Locumba evaluados en el presente estudio y determinado según los índices o unidades de productividad, se detallan en el Cuadro 2.

Cuadro 02: Aptitud acuícola de los recursos hídricos de la cuenca del río Locumba.

\begin{tabular}{|c|c|c|c|c|}
\hline$\omega^{*}$ & RECURSO HIDFUCO & $\begin{array}{l}\text { APTITUD } \\
\text { ACUICOLA }\end{array}$ & $\begin{array}{l}\text { RECURSO } \\
\text { HOROALLOEICO }\end{array}$ & $\begin{array}{l}\text { ACTMIDAD } \\
\text { ACUICOLL }\end{array}$ \\
\hline , & Quotrado Honda & & Trucha & $\begin{array}{l}\text { Repoblamiento, } \\
\text { cellivo }\end{array}$ \\
\hline 2 & $\begin{array}{l}\text { Rio euribaye } \\
\text { (chintorit) }\end{array}$ & & Camarón, trucha & $\begin{array}{l}\text { Repoblamiento, } \\
\text { cultoro }\end{array}$ \\
\hline 3 & Rlo Muaytite: & & Trueha & $\begin{array}{l}\text { Repoblamiento, } \\
\text { cultro }\end{array}$ \\
\hline 4 & Rro Tteatoye & & Trucha & $\begin{array}{l}\text { Repoblamianto: } \\
\text { cultivo }\end{array}$ \\
\hline$s$ & $\begin{array}{l}\text { procosunton } \\
\text { (puotion) }\end{array}$ & & Camaron & $\begin{array}{l}\text { Repobiampento. } \\
\text { cultivo }\end{array}$ \\
\hline - & IRlo llobeys & Mediane & Camarón, tructia & $\begin{array}{l}\text { Repoblamionto; } \\
\text { eutevo }\end{array}$ \\
\hline 7 & $\begin{array}{l}\text { Rlo cambingen } \\
\text { (s.pedro) }\end{array}$ & Medians & Trucha & $\begin{array}{l}\text { Repobilamiento. } \\
\text { cultivo }\end{array}$ \\
\hline 1 & 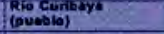 & Mediene & Truchs & $\begin{array}{l}\text { Repoblamiento, } \\
\text { culturo }\end{array}$ \\
\hline - & Rio Saledo & Modlana & Trucha & $\begin{array}{l}\text { Repoblamiento- } \\
\text { cultivo }\end{array}$ \\
\hline 10 & Choultepulo & Baja & Trucha & Cultivo, reproducción \\
\hline 11 & Rio calliasme (Aricoto) & Baja & Trucha & $\begin{array}{l}\text { Ropoblamiento, } \\
\text { cultivo }\end{array}$ \\
\hline
\end{tabular}

En la cuenca del río Locumba, el recurso hídrico conocido como Quebrada Honda, el río Curibaya (en la zona de Chintari), El río Tacalaya y el río Locumba (en la zona inmediata a la localidad del mismo nombre) son los que presentan la más alta productividad natural por la buena calidad de sus aguas, catalogándose a todos ellos con una aptitud acuicola óptima para el 
desarrollo de programas truchícolas de tipo extensivo e intensivo.

\section{Potencial o Aptitud Acuícola de la Cuenca del Rio Caplina-Uchusuma}

El potencial o aptitud acuícola de los recursos hídricos de la cuenca del río Caplina-Uchusuma evaluados en el presente estudio y determinado según los indices o unidades de productividad, se detallan en el Cuadro 3.

Cuadro 03: Aptitud acuícola de los recursos hídricos de la cuenca del río CaplinaUchusuma.

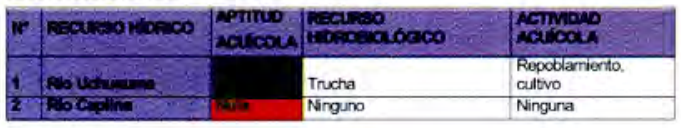

En esta cuenca, el único recurso hídrico que presenta buenas condiciones para la implementación de programas acuicolas orientados al repoblamiento y cultivo de trucha arco iris es el rio Uchusuma en la zona de Vilavilani. El río Caplina, por la baja calidad de sus aguas ( $\mathrm{pH}$ ácido), no alberga organismos acuáticos y, por consiguiente, su aptitud acuícola es nula.

\section{Potencial o Aptitud Acuícola de la Cuenca del Río Maure}

El potencial o aptitud acuícola de los recursos hídricos de la cuenca del río Maure evaluados en el presente estudio y determinado según los índices o unidades de productividad, se detallan en el Cuadro 4.

Cuadro 04: Aptitud acuícola de los recursos hídricos de la cuenca del río Maure.

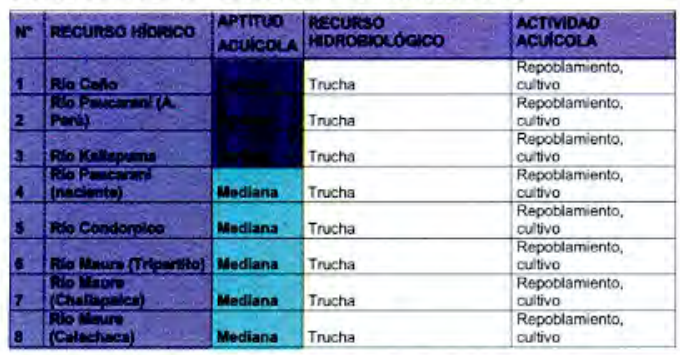

El río Caño, el río Paucarani en las proximidades de la localidad de Alto Perú y el rio Kallapuma, por presentar todos ellos una buena productividad natural, se les cataloga como de óptima aptitud acuícola y se puede establecer programas de repoblamiento $y$ cultivo de trucha arco iris.

El rio Paucarani cerca de su naciente, el río Condorpico y el río Maure en la mayor parte de su extensión, tienen una productividad natural moderada, presentando, por consiguiente, una mediana aptitud acuícola para programas truchícolas.
5. Potencial o Aptitud Acuícola de los Recursos Lénticos de Tacna

El potencial o aptitud acuícola de los recursos lenticos (lagunas y represas) de la región Tacna evaluados en el presente Estudio y determinado según los índices o unidades de productividad, se detallan en el Cuadro 5.

Cuadro

05: Aptitud acuícola de las lagunas y represa de la región Tacna.

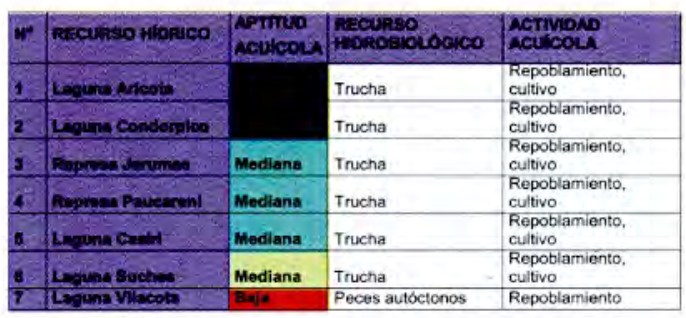

Las lagunas Aricota y Condorpico, por su buena productividad natural, presentan una óptima aptitud acuícola para el incremento de programas acuícolas orientados básicamente al cultivo extensivo (repoblamiento) y semiintensivo (jaulas flotantes) de trucha arco iris. Las represas de Jarumas y Paucarani y las lagunas de Casiri y Suches presentan una productividad natural moderada y, por lo tanto, su aptitud acuícola es mediana, también orientada al cultivo de trucha. La aptitud acuícola de la laguna Vilacota es baja por su someridad, a pesar de tener una productividad natural relativamente alta (gran cantidad de zooplancton, especialmente cladóceros); sin embargo, puede ser utilizada para programas experimentales de cultivo extensivo de especies autóctonas de la zona altiplánica, tales como el "suche", "carachi", "bagre", etc.

\section{CONCLUSIONES}

1.La mayoria de recursos hídricos de la región Tacna presentan buenas posibilidades para la implementación de programas acuícolas (cultivo de camarón de río y de trucha arco iris). Los únicos recursos hídricos en los cuales no es posible el establecimiento de estos programas son los rios Tarucachi y Caplina, por la mala calidad de sus aguas, especialmente por su acidez ( $\mathrm{pH}$ menos de 4,5) y la laguna Vilacota, por ser un ambiente léntico muy somero.

2.El potencial o aptitud acuícola de los recursos hídricos de las 04 cuencas hidrográficas y ambientes lénticos evaluados, presenta la siguiente categorización acuícola:

Buena aptitud acuícola : Cuenca del río Sama : ríos Sama, Ticalaco y Salado; cuenca del río Locumba: Quebrada Honda, ríos Curibaya, Huaytire, Tacalaya, Locumba, Callazas (San Pedro) y Salado (Aricota); cuenca del río 
Caplina-Uchusuma: únicamente el río Uchusuma; Cuenca del río Maure: ríos Caño, Paucarani, Kallapuma, Condorpico y Maure; laguna y represas: Lagunas Aricota, Condorpico, Camiri y Suches y represas Jarumas y Paucarani.

Baja aptitud acuícola: cuenca del río Sama: ríos Pistala y Chucatamani; Cuenca del río Locumba: Manantial Chaullapujo y río Callazas inmediatamente antes de su desembocadura en la laguna Aricota;lagunas: Laguna Vilacota.

Nula aptitud acuícola: Cuenca del río Sama: río Tarucachi; cuenca del río Caplina-Uchusuma: río Caplina.

\section{RECOMENDACIONES}

Es conveniente la ejecución de un programa de monitoreo permanente en los ríos y lagunas de la Región Tacna, desde el punto de vista limnológico y pesquero, para lo cual podría establecerse una Estación Limnológica en la Laguna Suches y otra en la Represa de Jarumas.

Las entidades involucradas en la captación y uso del recurso agua en la región (Proyecto Especial Tacna y Gobierno Regional de Tacna) deben considerar la ejecución de estudios tendentes a determinar el impacto ambiental y ecológico que pueda producirse como consecuencia del drenaje de lagunas, transferencia de recursos hídricos a otras cuencas y, en general, de las obras de ingeniería del afianzamiento de la Laguna de Aricota.

\section{BIBLIOGRAFIAA}

Chevarria, Angel y Coronel, Nelver. (1980). Inventario y evaluación de recursos hídricos continentales con fines piscícolas, Tacna Moquegua. ORDETAM-Dirección Regional de Pesquería, Tacna.

Clarke, George. (1994).Elementos de Ecología. Edic. Omega, S.A: Madrid.

Coronel, Nelver. (1982).Producción de truchas en jaulas flotantes - Estudio definitivo. ORDETAM, Dirección Regional de Pesqueria, Tacna.

Coronel F., Nelver (2005). Estudio bioecologico del río Sama, Perú. Tacna, 46 pp.

Huet, Marcel. (1983). Tratado de Piscicultura. Ediciones Mundi-Prensa, $3^{\text {a }}$. edición, Madrid.

Menacho, Guillermo. (1979). Campaña de siembra de alevinos de trucha en el Departamento de Moquegua. CODEMO, Moquegua.

Moreno, Tomás y Coronel, Nelver. (1987).
Estudio posibilidad de introducción de especies nativas. Micro region Tarata. Univ. Nac. Jorge Basadre Grohmann. Tacna.

Morris A., María Panty, Óscar. (1999). Espacio y conciencia geográfica en Tacna. ediciones Tercer Milenio, $1^{\mathrm{a}}$ edición, Tacna, 296 pp.

Needham, J.G. and Needham. (1962). A guide to the study of Freshwater Biology. Holden-Day. Inc. San Francisco. USA.

Odum, Eugene. (1972). Ecología. Edit. Interamericana, México.

Oikos S.R. Ltda. (1994). Programa de adecuación y manejo ambiental (PAMA) del cultivo extensivo de trucha en la laguna Suches, Tacna.

Oikos S.R. Ltda. (1995). Estudio limnológico y capacidad ictiológica de la Laguna Suches, Tacna.

\section{ANEXO}

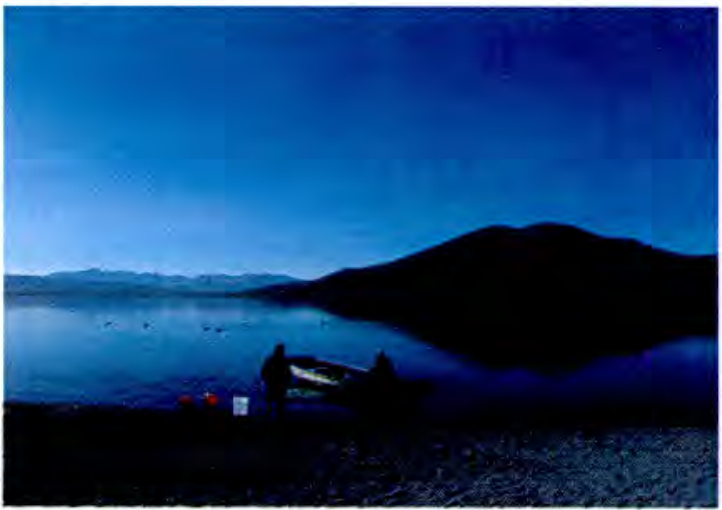

Foto 1: Laguna Suches, principal recurso léntico de Tacna.

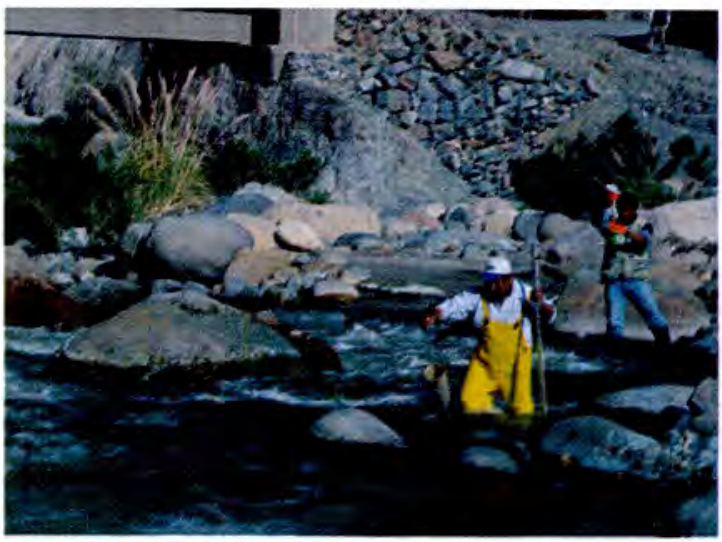

Foto 2: Evaluación limnológica en ríos de Tacna. 